\title{
TMS and tDCS in post-stroke aphasia: Integrating novel treatment approaches with mechanisms of plasticity
}

\author{
Jose Torres $^{\mathrm{a}}$, Daniel Drebing ${ }^{\mathrm{a}, \mathrm{b}}$ and Roy Hamilton ${ }^{\mathrm{a}, \mathrm{b}, *}$ \\ ${ }^{a}$ Department of Neurology, University of Pennsylvania, Philadelphia, PA, USA \\ ${ }^{\mathrm{b}}$ Laboratory for Cognition and Neural Stimulation, University of Pennsylvania, Philadelphia, PA, USA
}

\begin{abstract}
Aphasia is a common result of stroke, affecting over one million Americans. Currently, intensive speech therapy is the mainstay of treatment, although its efficacy has been variable at best. Recent years have seen the emergence of non-invasive brain stimulation, specifically Transcranial Magnetic Stimulation (TMS) and Transcranial Direct Current Stimulation (tDCS), as potential treatments for post-stroke aphasia. A growing body of investigations has shown the efficacy of both modalities in facilitating recovery from chronic aphasia, while data regarding subacute aphasia are much more limited and evidence in the acute post-stroke phase are still lacking. Much remains unknown about how these techniques cause clinical improvement or about their long-term efficacy, side-effects, and safety. In this article, we examine the data demonstrating the safety and efficacy of TMS and tDCS, discuss the major differences between them, and consider how those differences may inform the use of each modality. We also consider the different models of neuroplasticity in the setting of post-stroke aphasia and how these models may influence when and in which patients each modality would impart the most benefit.
\end{abstract}

Keywords: Transcranial Magnetic Stimulation (TMS), Transcranial Direct Current Stimulation (tDCS), aphasia, neuroplasticity, stroke

\section{Introduction}

Approximately seven million Americans over the age of twenty have had a stroke, and each year an additional eight hundred thousand experience a new or recurrent stroke. Of these, fifteen to thirty percent are permanently disabled (Roger et al., 2012). Aphasia is a common result of stroke, affecting an estimated $20 \%$ of patients (Roger et al., 2012). In over $80 \%$ of patients, post-stroke aphasia is due to infarction of

\footnotetext{
*Corresponding author: Roy H. Hamilton, MD, MS, Center for Cognitive Neuroscience, 518 Goddard Building, 3710 Hamilton Walk, University of Pennsylvania, Philadelphia, PA 19104, USA. Tel.: +1 215573 7065; Fax: +1 215573 7136; E-mail: roy.hamilton@uphs.upenn.edu.
}

the perisylvian regions of the left hemisphere and is initially marked by impairment or even total loss of language production, comprehension, or both (Alexander, 1997). Although some degree of language recovery over time is common, mostly occurring within the first three to six months after stroke, severe and debilitating language deficits frequently persist (Schlaug et al., 2009). Neuroimaging studies have demonstrated that the improvements that do occur rely on considerable changes in the cortical representation of language processing (Horn et al., 2005, Turkeltaub et al., 2011). To that end, evidence has pointed to at least two broad categories of functional change, namely recruitment of cortical areas surrounding the infarct, as well as activation of homotopic (analogous in location) regions in 
the opposite hemisphere (Basso et al., 1989, Basso \& Marangolo, 2000, Cappa et al., 1997).

Speech therapy is currently the most commonly employed treatment modality for aphasia. However, while improvements have been associated with intensive therapy, this treatment approach is time consuming, costly, and produces inconsistent results (Bhogal et al., 2003). Encouragingly, there is increasing evidence that non-invasive brain stimulation, specifically Transcranial Magnetic Stimulation (TMS) and Transcranial Direct Current Stimulation (tDCS), can induce changes in cortical function that enhance the recovery of certain language abilities in patients with post-stroke aphasia (Baker et al., 2010, Barwood et al., 2010, Floel et al., 2011, Fridriksson et al., 2011, Hamilton et al., 2010, Monti et al., 2008, Naeser et al., 2005a, Naeser et al., 2005b, Weiduschat et al., 2011). These studies are consistent with other investigations that have shown that non-invasive brain stimulation can lead to significant improvement in other domains, such motor paresis (Fregni et al., 2005, Hummel et al., 2005, Khedr et al., 2005, Mansur et al., 2005), and visuospatial neglect. (Ko et al., 2008, Oliveri \& Caltagirone, 2006, Oliveri et al., 2000, Oliveri et al., 1999).

Despite this emerging body of evidence, questions remain about how these techniques induce long-term plastic changes and about their overall safety in patients with stroke and other forms of brain injury. Furthermore, data accumulated over the last two decades has delineated clear differences between TMS and tDCS that may influence the circumstances under which each treatment modality should be considered. Additionally, emerging theories about the mechanisms of neural recovery after stroke may inform decision-making with respect to which brain stimulation approach to use. In this article, we will review the growing body of data demonstrating the efficacy of TMS and tDCS in patients with chronic post-stroke aphasia, and also present a more limited body of data suggesting the efficacy of brain stimulation in patients with subacute aphasia. While there are no studies on acute aphasic patients, we will briefly discuss studies in patients with motor abnormalities after acute stroke that hint at the notion that similar approaches may someday prove useful for acute aphasia. We will also review the major physiologic and practical differences between the two modalities and discuss how these differences may inform which technique should be administered to patients in different circumstances.

\section{Basic mechanisms of TMS and tDCS}

TMS was first presented at the London Congress of the International Federation of Clinical Neurophysiology by Anthony Barker in 1985 (Rossini \& Rossi, 2007). It employs a strong electrical current (approximately $5000 \mathrm{amps}$ ) that passes from a bank of capacitors through a coil of copper wires held above the scalp in order to create a brief, time-changing magnetic field. The magnetic field painlessly penetrates the skull to a depth of approximately 1.5 to $3 \mathrm{~cm}$ and if the amplitude, duration, and direction are appropriate, induces an electrical current in the underlying neurons that is sufficient to depolarize neuronal membranes and generate action potentials (Rossi et al., 2009). Different shapes and sizes of the coils affect the focality of the magnetic field. Circular coils are less focal, as their induced current is highest at the circumference of the coil with no current induced in the center. Figureof-eight coils are more focal, with the highest current induced at the intersection of the two round coils (Hallett, 2007). TMS pulses can be administered in a variety of different temporal patterns, including but not limited to single pulses, closely timed paired pulses, and trains of repetitive TMS (rTMS) pulses. Of these, rTMS has been the approach most frequently explored in stroke therapy. When administered at low frequencies (e.g. $1 \mathrm{~Hz}$ ), rTMS is believed to inhibit cortical excitability. Conversely, when administered at higher frequencies (e.g. $\geq 5 \mathrm{~Hz}$ ), rTMS can cause cortical excitation (Hallett, 2007). Both effects have been noted to persist after the discontinuation of stimulation (Barwood et al., 2010, Hamilton et al., 2010, Naeser et al., 2005a, Naeser et al., 2005b).

The use of tDCS in clinical practice dates back to the 1800s when Giovanni Aldini used direct stimulation of the brain to treat patients with mood disorders (Parent, 2004), and has re-emerged in recent years as an investigational tool and potential therapy (Nitsche \& Paulus, 2000). This method entails the application of small currents (typically 1-2 mA) to the scalp for a few minutes through two surface electrodes (usually $5 \times 5 \mathrm{~cm}$ or $5 \times 7 \mathrm{~cm}$ in size) soaked in isotonic saline (Nitsche et al., 2008, Nitsche \& Paulus, 2000). A common electrode configuration is a bipolar arrangement in which an active electrode is placed over the region of interest and a reference electrode is positioned over the contralateral supraorbital region; the reference electrode can also be placed over other sites that are relatively distant from the brain, such as over the mastoid or on 
the shoulder (Datta et al., 2008). Different electrode configurations are believed to affect the focality of stimulation and path of current flow. Recently, highdefinition tDCS systems have been introduced, which purport to increase the spatial resolution of stimulation by placing a smaller active electrode within a ring of reference electrodes (Datta et al., 2008). The electrical currents of tDCS are believed to modulate neuronal resting membrane potentials. This occurs in a bidirectional radial manner underneath the electrode and bidirectional tangential manner between the electrodes (Miranda et al., 2013, Peterchev et al., 2012). Unlike TMS, the currents generated using tDCS are believed to be insufficient to directly induce action potentials. Cathodal tDCS is associated with decreased cortical excitability due to subthreshold incremental hyperpolarization of membrane potentials, whereas anodal tDCS in believed to lead to increased cortical excitability due to incremental depolarization of membrane potentials. These effects may last minutes to hours after stimulation depending on the intensity, polarity, and duration (Nitsche et al., 2008), and repeated tDCS sessions can induce plastic changes in performance that can last for months (e.g. Reis et al., 2009).

\section{Models of neuroplasticity in aphasia recovery}

The therapeutic effects of TMS and tDCS in aphasic patients are believed to stem from the ability of these techniques to induce beneficial neuroplastic changes during the post-stroke reorganization of language areas (Horn et al., 2005, Turkeltaub et al., 2011). Converging evidence indicates that there is increased activation in remaining perilesional regions of the dominant (left) hemisphere, as well as in regions of the nondominant (right) hemisphere homotopic to normal language areas, in patients with post-stroke aphasia (Turkeltaub et al., 2011). However, the role of the right hemisphere in language recovery has been controversial, giving rise to three basic models of neuroplasticity as it relates to aphasia recovery: 1) beneficial recruitment of perilesional areas surrounding the infarcted tissues in the dominant hemisphere, 2) beneficial recruitment or disinhibition of homotopic language areas in the nondominant hemisphere and 3) activation of inefficient or deleterious language centers in the right hemisphere that may interfere with recovering language networks (Fig. 1) (Hamilton et al., 2011, Heiss \& Thiel, 2006,

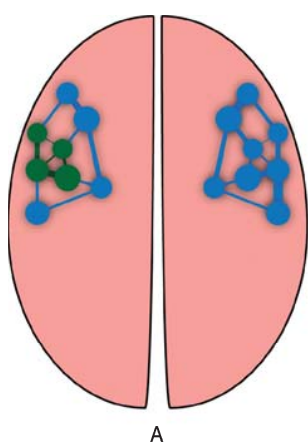

Normal Functioning

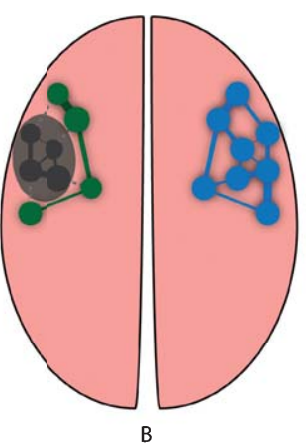

Perisylvian Recruitment

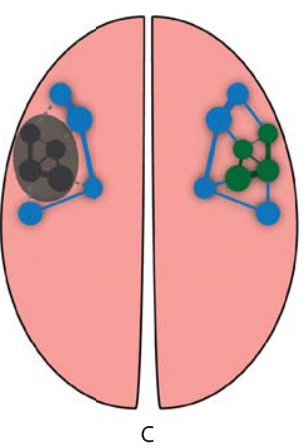

Homotopic Recruitment

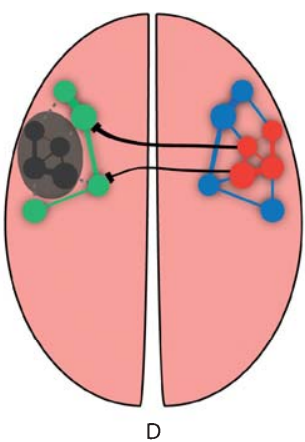

Detrimental Inhibition

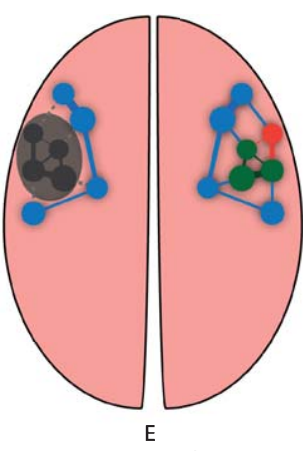

Noisy Nodes

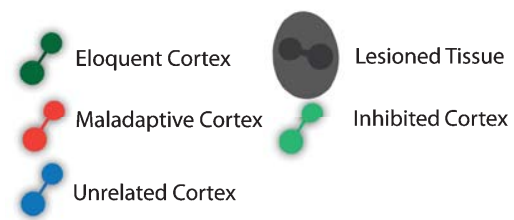

Fig. 1. Proposed neuroplastic mechanisms for recovery from aphasia. A. In the normal state, language is mediated by a perisylvian network of brain regions in the left hemisphere. B. Typical language network areas may be destroyed by stroke (shaded), but perilesional areas may reorganize to subsume language functions. C. After a left hemisphere stroke, homotopic areas in the right hemisphere may be recruited or disinhibited to perform language functions. D. Perilesional areas may reorganize to perform language functions, but may be subject to heightened right hemisphere inhibition of the left hemisphere. E. Homotopic areas in the right-hemisphere may reorganize to perform language functions, but some areas (highlighted in red) could potentially act inefficiently (so-called "noisy nodes"), and may thus be detrimental to overall functioning. 
Thiel et al., 2006, Turkeltaub et al., 2012, Turkeltaub et al., 2011).

The recruitment of perilesional areas is thought to result from the release of cortico-cortical inhibitory inputs from infarcted language centers (Shimizu et al., 2002). It is generally agreed upon that the contribution of the left hemisphere perilesional areas to aphasia recovery is beneficial in nature. Consistent with this belief, several studies have shown that patients with greater left hemispheric activation after stroke have more spontaneous recovery of speech (Karbe et al., 1998, Warburton et al., 1999) and better language performance after speech therapy (Cornelissen et al., 2003, Leger et al., 2002).

Post-stroke neuroplasticity also involves the recruitment of right-sided homotopic areas that may compensate for damaged left-sided language centers. This model has been supported by neuroimaging studies of post-stroke aphasic patients. For example, in an Activation Likelihood Estimation (ALE) metaanalysis of published functional imaging (fMRI) data involving chronic aphasic patients and healthy controls, our group has demonstrated that patients with lesions involving the left inferior frontal gyrus (IFG) activate a bilateral network of cortical areas during language tasks, which includes a number of regions in the right hemisphere that are homotopic to perisylvian left hemisphere language centers. Moreover, further analysis suggested that many, but not all, of these areas are homologous in function to their left hemisphere counterparts in normal individuals, further supporting the compensatory role of the right hemisphere in stroke recovery (Turkeltaub et al., 2011).

However, the role of the non-dominant hemisphere in aphasia recovery remains controversial, as some studies, including our own, have actually shown improved language after inhibition of the right IFG using low-frequency rTMS (Barwood et al., 2010, Hamilton et al., 2010, Medina et al., 2012, Naeser et al., 2010, Naeser et al., 2005a, Naeser et al., 2005b). There are at least two theories that attempt to explain these discordant data. One of these is based on the existence of inhibitory connections between the two hemispheres. By this account, left-sided strokes disrupt homeostatic competition, resulting in unopposed inhibition of the left hemisphere by the right. Right hemisphere over-activity, in turn, inhibits compensatory perilesional left hemisphere activity (Crosson et al., 2007). According to this model, inhibitory right hemisphere non-invasive brain stimulation can be used to improve language function by decreasing this excessive activity and releasing left hemisphere perisylvian areas from inhibition (Fregni et al., 2005). This theory is consistent not only with prior brain stimulation studies, but also with functional imaging data that suggest that activation of right hemisphere areas during language tasks is, at least in some cases, linked with worsened performance (Postman-Caucheteux et al., 2010).

While the inter-hemispheric inhibition model may provide a possible explanation for the beneficial effects of inhibitory brain stimulation on language, this account does not explain other findings. For instance, we and others have found that the beneficial effects of inhibitory rTMS in patients with chronic aphasia are topographically specific, with the majority of patients responding positively to stimulation of a single region, the right pars triangularis (Hamilton et al., 2010, Medina et al., 2012, Naeser et al., 2005b). We have argued that this degree of specificity does not support the notion that the right hemisphere generally plays a deleterious role in aphasia recovery. Rather, we have argued that in many patients with aphasia, the right hemisphere contributes beneficially to a reorganized language network supporting functional recovery, but that certain sites ("noisy nodes") in that network may not contribute to that network efficiently. This theory is supported by our prior ALE analysis (Turkeltaub et al., 2011), in which the right pars triangularis was shown to be one unique site that was activated during language tasks, but was not homologous in function compared to its left hemisphere counterpart in normal individuals. Moreover, the notion that parts of the right hemisphere can be beneficial to language recovery while others are detrimental is supported further by a case we reported (Turkeltaub et al., 2012), in which a patient with chronic aphasia showed improvement in language functions after receiving inhibitory rTMS of the right pars triangularis, only to experiencing significant and selective worsening of her aphasia after suffering a subsequent stroke of the right hemisphere.

Other important variables that influence the neuroplastic mechanisms of aphasia recovery are lesion size and stroke chronicity. It has been argued that stroke size may be critical for determining the nature of inter-hemispheric interactions in aphasia recovery. According to this account, small strokes leave critical language centers of the dominant hemisphere largely intact, such that recruitment of perilesional areas may be sufficient for significant language recovery. In this 
setting, the increased activation of right-sided homotopic regions may be more likely to inhibit the function of these left-sided language centers and have a deleterious effect on recovery (Schlaug et al., 2011, Thiel et al., 2006, Warburton et al., 1999). By contrast, in the case of large left-sided strokes, there may not be sufficient healthy left hemispheric perilesional tissue to recruit for language processing and, as a result, the intact right hemisphere must assume a greater role in language processing (Rosen et al., 2000, Schlaug et al., 2011, Thiel et al., 2006). While these areas may not be as efficient as left-sided language areas in language processing, they are, nevertheless, compensatory, and their disruption could lead to loss of recovered function.

Another possibility may be that different regions are recruited at different times during the recovery process (Saur et al., 2006). According to this account, acutely after a stroke, when there is damage to the left hemisphere and not enough time has elapsed to recruit homotopic regions in the right hemisphere, neither side subserves language processing. This is consistent with the observation that patients' language deficits are most severe during the acute phase. Subacutely, right-sided homotopic areas appear to take on some lost language functions. However, these regions do not appear to be as efficient as their left-sided counterparts, and activation of these regions is generally associated with more limited language recovery (e.g. Heiss \& Thiel, 2006, Postman-Caucheteux et al., 2010) Finally, over time, the left side may recover to the point that more efficient language centers may be able to reassert control over language function, producing further functional improvement (Saur et al., 2006).

Which of these models holds true remains unclear, and further research will be needed to fully characterize the neuroplastic changes that occur in post-stroke aphasia. However, in recent years, several studies have demonstrated that both TMS and tDCS can be used to alter brain neuroplasticity and enhance the rehabilitation of aphasic patients.

\section{Clinical studies of TMS in aphasia and other post-stroke deficits}

Most studies utilizing TMS in aphasia have been based on the assumption that right hemispheric activation may impair language recovery, and therefore have predominantly used inhibitory rTMS to right-sided homotopic areas. Also, most studies have focused on patients with chronic post-stroke aphasia (Barwood et al., 2010, Hamilton et al., 2010, Naeser et al., 2005a, Naeser et al., 2005b). Here the work of Naeser and colleagues has been highly influential. In 2002, this group applied inhibitory rTMS during one 10-minute session to the pars triangularis (PTr) of six chronic non-fluent aphasic patients and showed significant improvement in naming accuracy and reaction time, though this improvement lasted less than 30 minutes. They also noted that inhibitory stimulation of the pars opercularis (POp) actually worsened performance (Naeser et al., 2002). In a follow up study, the same group applied $1 \mathrm{~Hz}$ rTMS for 20 minutes a day for 10 days to the PTr of 4 chronic aphasic patients and found that at two months post-stimulation, there were significant improvements in the first 20 items of the Boston Naming Test as well as the Tools/Implements measure and Animal Naming subsets of the Boston Diagnostic Aphasia Exam (BDAE). At eight months, there was still continued improvement in these language tests, but only the Tools/Implements testing was statistically significant, and while there was also improvement in the phrase length of elicited propositional speech, this was sustained at two months but not at eight months (Naeser et al., 2005b).

In an effort to better establish the site-specific effects of inhibitory stimulation, Naeser and colleagues examined how a 10-minute session of $1 \mathrm{~Hz}$ rTMS affected picture naming in an additional eight chronic aphasic patients and eight normal controls. They specifically examined the immediate effect on naming after stimulation of the PTr, POr, motor cortex mouth area (M1), and the posterior-superior temporal gyrus (STG; Wernicke's homologue region) and found significant improvement in the number of pictures named and reaction time in the aphasic patients after inhibitory rTMS of the PTr but an increase in reaction time after stimulation of the POp. Additionally, there was a decrease in reaction time with M1 stimulation but no change in the number of pictures named and there was a decrease in pictures named with STG stimulation but no change in reaction time. While normal controls were able to name all pictures, reaction time decreased significantly with right versus left PTr inhibition and increased significantly with left versus right POp inhibition (Naeser et al., 2011).

Additional studies have confirmed these results. We found similar evidence, noting that in a patient with chronic aphasia, inhibition of the PTr resulted in a $41 \%$ increase in naming ability, whereas inhibition 


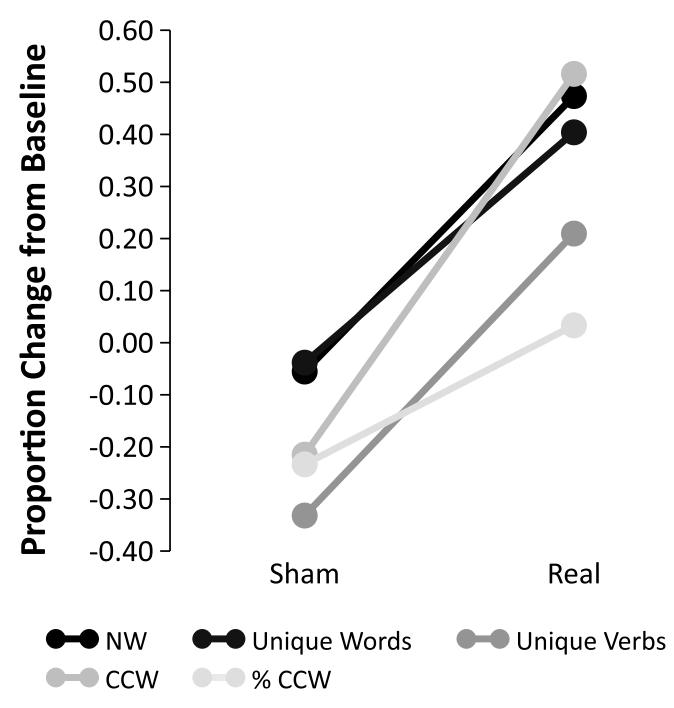

Fig. 2. rTMS improved spontaneous speech in patients with chronic nonfluent aphasia (Modified from Medina et al., 2012). After two weeks (M-F) of 20-minute sessions of $1 \mathrm{~Hz}$ rTMS delivered to the right inferior frontal gyrus, aphasic patients showed improvement in multiple measures of spontaneous speech compared to subjects receiving sham stimulation. The figure shows the proportion change from baseline in discourse productivity for subjects tested at 2 months following sham and real rTMS on the Cookie Theft Picture Description from the Boston Diagnostic Aphasia Examination (BDAE; Goodglass, Kaplan, \& Barresi, 2001). Measures employed were taken from Quantitative Production Analysis (QPA; Rochon, Saffran, Berndt, \& Schwartz, 2000); the specific measures shown were those in which subjects receiving real stimulation exhibited significant improvement compared to baseline. $\mathrm{NW}=$ narrative words; $\mathrm{CCW}=$ closed-class words; OCW = open class words.

of the nearby anterior POp and ventral-posterior PTr resulted in a $23 \%$ decrease in naming (Hamilton et al., 2010). In a follow-up study, we investigated the long-term response to 10 days (TMS Mon-Fri for two weeks) of 20-minute session of $1 \mathrm{~Hz}$ rTMS in a cohort of 10 subjects with chronic nonfluent aphasia, and found lasting improvement in naming and multiple measures of spontaneous speech (Medina et al., 2012, Fig. 2). As with prior studies by Naeser and colleagues (2005b, 2011), we determined the optimal site for stimulation by briefly targeting a variety of sites in the right IFG with a 10-minute session of $1 \mathrm{~Hz}$ rTMS. Supporting prior data indicating the topographic specificity of rTMS, we found that nine out of 10 subjects demonstrated the largest transient increase in naming performance following stimulation of the right PTr. Similarly, Barwood and colleagues applied $1 \mathrm{~Hz}$ rTMS to the right PTr of six non-fluent aphasic patients who were two to six years post stroke. After ten 20-minute sessions, they found significant improvements in picture naming, spontaneous speech output, and auditory comprehension at two months, when compared to aphasic patients receiving sham treatment (Barwood et al., 2010). They also examined treatment-related modulation of N400 event-related brain potentials, which has been associated with lexical and semantic processing and found sustained modulation at two months after stimulation (Barwood et al., 2011). More recently, they looked at seven non-fluent chronic aphasic patients and examined the effects of inhibitory stimulation to the right PTr over ten 20-minute sessions. They noted improved picture naming, spontaneous speech, naming accuracy, and auditory comprehension tasks including complex ideational materials and complex commands at one week, two months and eight months (Barwood et al., 2012).

While most investigators have employed inhibitory stimulation of the intact hemisphere, Szaflarski and colleagues have used excitatory stimulation to the lesioned hemispheres of chronic aphasic patients to induce neuroplastic changes. In a 2011 paper, they examined eight patients who underwent ten 200second sessions of excitatory, $50 \mathrm{~Hz}$ rTMS to the left Broca's area. They noted that after stimulation, six of eight patients showed significant improvement in semantic fluency and were able to generate more appropriate words when prompted with a semantic category. They also found that there was increased fMRI activity in the left fronto-temporo-parietal language areas during language tasks when compared to functional imaging prior to stimulation as well as a significant shift in activity to the left side during language tasks (Szaflarski et al., 2011).

Similar improvements have also been demonstrated in patients with subacute aphasia. Weiduschat and colleagues examined the effects of inhibitory rTMS to the right IFG of six aphasic patients. The patients also received speech and language therapy immediately after stimulation. Using the Aachen Aphasia Test to assess language performance, the investigators found that compared to the sham group, the stimulation group's score improved by an average of 11.3 points. Moreover, they assessed language area activation using PET scans and found that the treatment group was less likely to lateralize language to the right side (Weiduschat et al., 2011).

While there have been no rTMS studies on aphasic patients in the acute post-stroke phase, there have been 
studies looking at the efficacy of rTMS in patients with acute hemiparesis after stroke. For example, Khedr and colleagues studied 52 right-handed patients within two weeks of a first non-hemorrhagic stroke. Of these, 26 patients received excitatory rTMS over the lesioned hemisphere and an equal number received sham treatment. Of these, the treatment group had a higher percentage of independent patients and lower stroke scales (Khedr et al., 2005). In another study, the same group showed that $1 \mathrm{~Hz}$ rTMS to the non-lesioned hemisphere and $3 \mathrm{~Hz}$ rTMS to the lesioned hemisphere induced significant improvements in Pegboard tasks and keyboard typing at three months as compared to the sham group, though the $1 \mathrm{~Hz}$ group performed better (Khedr et al., 2009). Several additional studies have confirmed that rTMS may be a promising potential intervention in patients with acute motor weakness (see Ayache, 2012 for a review). Thus, while acute studies of aphasia are lacking, evidence suggests that rTMS studies are feasible in the acute post-stroke phase, and that rTMS administered acutely can facilitate improvement of other types of neurologic deficits. Future investigations will need to determine whether rTMS can be of similar benefit to patients with acute post-stroke aphasia.

\section{Clinical studies of tDCS in aphasia and other post-stroke deficits}

Compelling evidence also exists for the efficacy of tDCS in modulating and enhancing the post-stroke recovery of aphasic patients. Monti and colleagues were the first to use tDCS along with speech therapy in the rehabilitation of aphasic patients. They administered either sham stimulation or anodal/cathodal stimulation to eight chronic aphasic patients with stimulation consisting of one 10-minute session of $2 \mathrm{~mA}$ to the left fronto-temporal region. They found that cathodal stimulation significantly improved naming accuracy by a mean of $33.6 \%$, whereas anodal and sham stimulation had no effect. This result was somewhat unexpected given the theories of neuroplasticity discussed above. The authors suggested that the improvement seen with cathodal stimulation to the lesioned hemisphere may have been the result of decreased activity of overactive inhibitory interneurons in the left hemisphere, allowing for better compensation by the perilesional areas (Monti et al., 2008).
Arguing that the null result with anodal stimulation in the Monti study might be related to identical placement of electrodes irrespective of aphasia subtype and of individual differences in the reorganization of language representation between patients, Baker and colleagues instead used fMRI data to guide electrode placement and tested only chronic non-fluent aphasic patients. They examined the naming ability of 10 chronic aphasic patients after five 20-minute sessions of $1 \mathrm{~mA}$ anodal stimulation administered concurrently with computerized anomia training and found significant improvements in naming after stimulation when compared to pre-stimulation testing. In a followup crossover study, the same group recruited eight chronic, fluent aphasic patients and treated them with five 20-minute sessions of $1 \mathrm{~mA}$ anodal stimulation to the left hemisphere. They found that compared to sham treatment, there was reduced reaction time during picture naming immediately after the five sessions and at three weeks. Interestingly, there was an improvement in the naming of novel (unlearned) words, though this effect did not reach statistical significance, indicating that tDCS may not simply improve the naming of words that have been learned but may also have a more generalizable effect on lexical retrieval (Baker et al., 2010, Fridriksson et al., 2011). Similarly, Fiori and colleagues applied $1 \mathrm{~mA}$ stimulation to Wernicke's area of three aphasic patients as well as 10 healthy subjects. The healthy subjects were taught 20 new words during the sessions while the aphasic patients underwent intensive language training during stimulation. They found that after stimulation aphasic patients had improved picture-naming accuracy and both groups had improved naming latencies during anodic stimulation of Wernicke's area when compared to sham (Fiori et al., 2011).

Cathodal stimulation to the right side has also been shown to improve outcomes in aphasic patients. You and colleagues examined 33 subacute stroke patients with global aphasia using the Korean Western Aphasia Battery. Patients underwent speech therapy concurrently with either ten 30-minute sessions of $2 \mathrm{~mA}$ stimulation or sham stimulation. The treatment group was further divided into anodal stimulation of the left hemisphere or cathodal stimulation of the right hemisphere. The investigators found no significant difference in spontaneous speech, repetition, or naming among the three groups. However, they did find improvements in aphasia quotients and auditory verbal comprehension in the treatment groups, with these 
improvements most pronounced with cathodal stimulation of the right hemisphere (You et al., 2011). Similarly, in a cohort of 10 patients with chronic aphasia, Kang and colleagues applied five 20-minute sessions of $2 \mathrm{~mA}$ cathodal stimulation over the right IFG in combination with conventional word retrieval therapy and found improvement in naming accuracy on the Korean version of the Boston Naming Test (BNT) immediately after the five sessions (Kang et al., 2011).

At the present time, as with rTMS, no studies have examined the effects of tDCS in acute aphasic stroke patients. However, tDCS has been applied to patients with acute stroke-induced motor dysfunction. Here the results have been equivocal. For instance, Rossi and colleagues applied five 20-minute sessions of anodal stimulation to the affected motor cortex of 25 patients two days after their stroke. When compared to 25 patients who received sham treatment, they did not find any significant differences in Fugl-Meyer Assessments and NIH strokes scales between the two groups at five days and at three months after treatment (Rossi et al., 2013). However, other studies of the effects of tDCS on acute paresis have been more encouraging (see Ayache, 2012 for a review). As with rTMS studies in acute paresis (see above), these studies suggest that the experimental investigations involving tDCS in acute stroke are feasible. Clearly, further studies are needed to establish the role that tDCS may play in the treatment of acute aphasia.

\section{Cellular mechanisms of action}

Despite this growing literature on the potential benefits of rTMS and tDCS in the recovery of aphasic patients, the cellular mechanisms that drive behavioral plasticity in non-invasive brain stimulation have not been fully characterized. However, emerging evidence shows that these modalities cause biochemical changes similar to what is seen with long-term potentiation (LTP) and long-term depression (LTD). In the case of LTP, excitation of presynaptic neurons leads to glutamate release. Glutamate then binds AMPA [2amino-3-(5-methyl-3-oxo-1,2- oxazol-4-yl) propanoic acid] receptors on the surface of the postsynaptic neurons, leading to an influx of sodium and potassium and causing depolarization of the postsynaptic neurons (Thickbroom, 2007). Glutamate also binds NMDA [N-methyl-D-aspartic acid] receptors, which usually contain magnesium molecules that block calcium influx into postsynaptic neurons. However, if there is sufficient postsynaptic depolarization, this blockade is removed and calcium is able to enter the postsynaptic neurons (Hoogendam et al., 2010). Rapid increases in calcium influx lead to the auto-phosphorylation of AMPA receptors as well as insertion of additional AMPA receptors into the cell membranes of postsynaptic neurons (Thickbroom, 2007). This causes the postsynaptic cells to be more permeable to cations and more easily excitable. Conversely, a slow rise in calcium influx leads to the removal of AMPA receptors from the cell membranes, decreasing their excitability and leading to LTD (Thickbroom, 2007). Calcium influx also activates calcium-sensitive pathways that alter protein synthesis and gene expression in these neurons (Hoogendam et al., 2010).

TMS and tDCS are thought to invoke plastic change by instigating many of these same mechanisms. For instance, researchers have demonstrated increased levels of Zif268 and cFOS in rat brains that are stimulated by rTMS and tDCS (Aydin-Abidin et al., 2008, Ranieri et al., 2012). These proteins are known to be important in maintaining long-term synaptic connections and are increased by LTP (AydinAbidin et al., 2008). Gersner and colleagues have shown an increase in AMPA receptor phosphorylation was well as a significant increase in hippocampal BDNF (Brain-Derived Neurotrophic Factor) in rat brains after 13 days of rTMS (Gersner et al., 2011). BDNF is important in modulating synaptic strength (Hoogendam et al., 2010) and certain BDNF polymorphisms that are known to decrease BDNF activity are associated with memory problems and decreased hippocampal volume in humans. These polymorphisms have also been shown to inhibit cortical excitability after stimulation with TMS and tDCS (Cheeran et al., 2008), implying a relationship between cortical changes induced by these modalities and BDNF activity. Studies investigating the effects of drugs on the ability of TMS and tDCS to induce plastic changes have provided further insight into the cellular mechanisms of both modalities. NMDA receptor antagonists such as Memantine and Dextromorphan, have been found to mediate the effects of TMS and tDCS (Huang et al., 2007, Stefan et al., 2002), suggesting that the changes that occur with both rely on NMDA receptor activity. Also, calcium channel blockers such as Carbamazepine have been found to decrease the effects of anodal tDCS, demonstrating the importance of calcium 
pathways in tDCS-induced neuroplasticity (Nitsche et al., 2003).

Interestingly, Nitsche and colleagues have found that, unlike rTMS, neither cathodal nor anodal tDCS alters the motor thresholds required to induce muscle contractions with stimulation of the motor cortex (Nitsche et al., 2005). However, input/output curves, which reflect the influence of interneurons on cortical excitability, were modulated by cathodal tDCS (Nitsche et al., 2005), and intracortical facilitation (ICF), which is diminished by GABAergic substances and reflects the overall activity of GABAergic interneurons, was decreased by cathodal tDCS (Nitsche et al., 2005). Therefore, it appears that at a cellular level both rTMS and tDCS induce long-term potentiation and depression by altering protein synthesis necessary for strong synaptic connections. However, based on the work of Nitsche and colleagues, tDCS may affect these changes through the modulation of interneurons as opposed to the direct stimulation of neuronal networks.

\section{Important advantages and disadvantages of each modality}

Important practical differences exist between TMS and tDCS, which may make the use of one or the other technique more advantageous in specific situations. For instance, the temporal resolution of TMS is much greater than that of tDCS, since TMS pulses can be administered with millisecond-level precision. Related to this, because TMS pulses induce immediate action potentials, one can instantly see the short-term effects of stimulation, through measurement of transient changes in relevant perceptual, cognitive, or motor functions (Hallett, 2007). TDCS, on the other hand, modulates membrane potentials without depolarizing them, and stimulation must be administered over the course of minutes, leading to significantly lower temporal resolution (Nitsche et al., 2008).

Spatial resolution is also much higher with TMS. In tDCS, current flows from large electrodes into brain tissue (Datta et al., 2008). While it is generally assumed that most of the current administered remains underneath the active electrode, Bikson and colleagues have found that there is wide dispersion of current associated with the use of standard electrodes (Bikson et al., 2009). This may be advantageous when one wants to stimulate large areas of brain, as is the case when activating perilesional language areas surrounding left-sided strokes. However, it can also be an important disadvantage in situations where stimulation of neighboring structures can have very disparate effects on performance outcomes (Hamilton et al., 2010).

Differences in electrode placement can have significant effects on current distribution as well. Some investigators have found that placing the active electrode within a ring of reference electrodes can increase the focality of current (Bikson et al., 2010). However, the efficacy of this electrode setup is not yet clear. Also, Fridriksson and colleagues demonstrated that when the cathode is placed on the contralateral orbito-frontal scalp instead of the shoulder, there is less concentration of current in the perilesional areas (Fridriksson, 2011), possibly decreasing the positive effects of tDCS on these areas.

Not only is TMS stimulation more focal, but the region of stimulation is also presumably more predictable across different patients. In TMS, the magnetic field can penetrate the skull without much interference (Hallett, 2007). tDCS-administered current, on the other hand, must go through scalp and bone, which can prevent significant amounts of current from reaching brain tissue. Since individuals have unique bone thicknesses and amounts of hair, the current administered can vary significantly from patient to patient (Fridriksson et al., 2011). Furthermore, lesioned tissue may not behave the same as normal tissue with respect to the propagation of current. Fridriksson and colleagues have noted that current sometimes becomes sequestered within lesioned tissue instead of traveling to perilesional areas and in chronic stages of stroke, where lesions can become gliotic and filled with cerebrospinal fluid, the current can be dispersed by the fluid, preventing maximal stimulation of targeted tissues (Fridriksson et al., 2011). TMS is not without its drawbacks though. While it is presumed that TMS gets one around these problems, some variability in stimulation does occur because of differences in the distance between the scalp and brain tissue in patients (Stokes et al., 2007, Wagner et al., 2008), as well as differences in the flow of induced current in heterogeneous neural and non-neural elements (Wagner et al., 2006)

Portability is also a significant difference between the two modalities. While tDCS requires only electrodes and a small current stimulator that would fit inside a briefcase, TMS typically requires a large current generator, stimulation coils and, when a MRIguided neuro-navigational system is used, an infrared camera and localizing tools for use on the head of 
the subject and on the coil. The latter setup prevents patients from undergoing simultaneous physical therapy or any therapy that would involve considerable changes in position, which is problematic as some studies have shown that concomitant therapy may improve functional outcomes in stroke patients (Nitsche et al., 2003). Additionally, TMS is more expensive than tDCS. TDCS systems typically cost approximately ten to fifteen thousand dollars. A TMS system, on the other hand, can cost an order of magnitude more than this, particularly if an MRI-guided stereotactic system is used.

\section{Applying both modalities to the models of neuroplasticity}

Because of these differences between the two modalities, particularly in terms of spatial resolution, rTMS and tDCS could potentially be applied differently depending on which model of neuroplasticity one holds to be correct (Fig. 3). As explained above, one inter-hemispheric model supposes that dysfunctional inhibition from the non-lesioned hemisphere prevents the recruitment of perilesional areas in the dominant hemisphere and inhibits recovery of language function. In this situation, cathodal tDCS, which has low spatial resolution and the potential to incrementally suppress the entire right hemisphere, could theoretically be used to re-establish the functional balance between the two hemispheres. RTMS, on the other hand, may be too focal to optimally prevent transcallosal inhibition after a large lesion of the left hemisphere. Conversely, if one holds, as we have argued, that the right hemisphere generally contributes beneficially to a reorganized language network, but that certain centers ("noisy nodes") in that network may not contribute to that network efficiently, then focal inhibition of these "noisy nodes" would more effectively promote language recovery. In this case, tDCS might not be expected to achieve that goal as robustly as rTMS, owing to its lower topographic resolution.

If one argues that the size of the stroke is the most important factor in establishing whether the right side is detrimental or beneficial in the post-stroke recovery of aphasic patients, then based on the assumption that patients with small strokes preferentially recruit left hemisphere perilesional areas, one could use excitatory rTMS techniques to stimulate left hemisphere regions that on functional imaging are shown to be activated during language tasks. Alternatively, based on the notion that the right hemisphere is less likely to play a beneficial role in the setting of small left hemisphere lesions and left perilesional recruitment, one could apply cathodal tDCS to the right hemisphere, to prevent it from inhibiting the effective recruitment of these perilesional areas. Moreover, these interventions - targeted perilesional excitatory

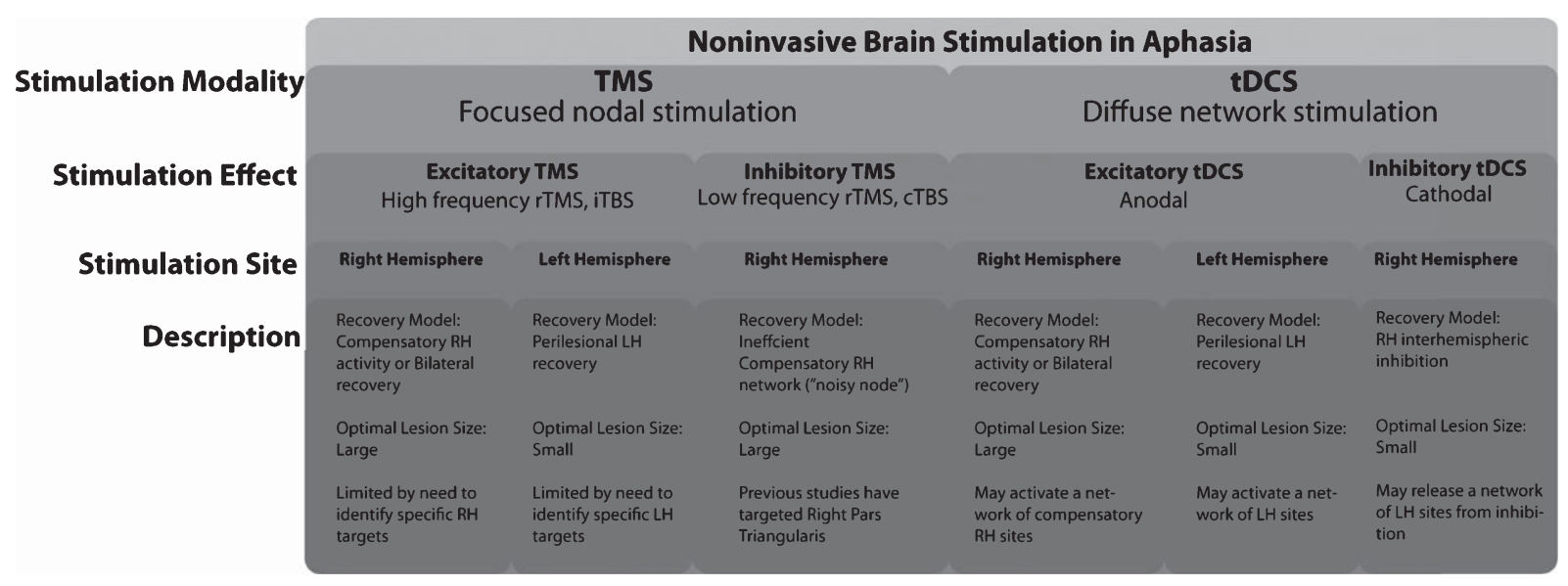

Fig. 3. Models of language plasticity inform stimulation approaches in aphasia therapy. Different models of neuroplasticity lend themselves to specific stimulation approaches, based on differing accounts of the contribution of the left and right hemispheres to recovery from aphasia. Proposed stimulation techniques generally entail either excitation of compensatory networks in either the left or right hemisphere or inhibition of inefficient or deleterious regions of the right hemisphere. Also, stroke size may inform whether the preferable stimulation approach is leftor right-sided, since large strokes may not allow for adequate compensatory changes to occur in the left hemisphere. 
TMS and right-sided inhibitory tDCS could potentially be combined. One could also concurrently apply anodal tDCS to the left hemisphere and cathodal stimulation to the right hemisphere to achieve similar effects. On the other hand, if the lesion is large, the appropriate assumption may be that activation of the right hemisphere is crucial to language recovery. In that case, anodal tDCS to a broad network of compensatory right hemisphere regions may be optimal.

Finally, because it may be the case that different patterns of neural activation are required to facilitate language performance at different stages of aphasia recovery, different TMS and tDCS approaches could potentially be effective at different points in time. For instance, at least some evidence suggests that the right hemisphere may be more beneficial in the subacute phase of stroke recovery, while left hemisphere perilesional activation is appears to be more robust in the chronic phase (e.g. Saur et al., 2006). By this account, one could conceivably argue for different approaches to stimulating the right hemisphere based on stroke chronicity, with anodal right hemisphere tDCS being useful for enhancing stimulation of a network of compensatory right hemisphere regions in the early period after stroke, while cathodal stimulation may be beneficial in the later phases, when left hemisphere recruitment appears to be more crucial. Additionally, insofar as the role of the left hemisphere perilesional areas is generally thought to be beneficial, one approach dictated by a dynamic model of right hemisphere function would be to focus on excitatory stimulation of left hemisphere sites, either using targeted excitatory TMS of functionally relevant perilesional areas or more distributed left hemisphere anodal tDCS.

Therefore, tDCS and rTMS could be used differentially depending on theoretical models of language neuroplasticity. Models that favor more topographically specific neuroplastic changes argue for the more targeted stimulation afforded by rTMS, whereas models that assert that broader hemispheric networks and their interactions are more important to language recovery potentially argue for the more distributed stimulation of tDCS. Clearly, in order to better inform decisions regarding the use of rTMS or tDCS in aphasia, additional studies are needed in order to better characterize the role of lesion size, lesion location, stroke chronicity, and a variety of other clinical factors that influence language recovery and response to brain stimulation.

\section{Safety of both techniques}

One last consideration which distinguishes rTMS from tDCS is the difference in safety concerns for each modality. For rTMS, an important potential concern is seizures, presumably related to the ability of TMS to synchronize neuronal discharges. The risk of seizure induction is very low; since 1998 there have only been nine cases of seizure reported during or immediately after rTMS despite the large number of patients that have undergone stimulation (Rossi et al., 2009). What's more, current guidelines set clear limits with respect to stimulation frequency, intensity, number of pulses, and inter-stimulus interval, within which the use of rTMS is considered to be very safe (Rossi et al., 2009).

Meta-analyses have confirmed the safety of these guidelines. Machii and colleagues reviewed side-effect data from January 1998 to December 2003 in studies that applied rTMS to non-motor areas. In this study encompassing over 1,100 patients, they found that headache was the most common side-effect, occurring in $23 \%$ of patients. Serious side-effects were rare, with two documented seizures and four episodes of psychosis (Machii et al., 2006). Janicak and colleagues examined data on 325 patients who underwent a combined 10,000 rTMS sessions for major depressive disorder. They noted that most side-effects were mild to moderate in intensity with scalp discomfort and transient headaches being the most common. Only 4.5 $\%$ of patients discontinued the sessions because of side effects (Janicak et al., 2008). Even studies looking at the effects of TMS on seizure patients have found that TMS is safe in this population, noting an associated seizure risk of 0 to $2.8 \%$ for single pulse TMS and 0 to $3.6 \%$ for paired pulse TMS (Schrader et al., 2004).

Another concern is in patients with intracranial or peri-cranial implants and metals such as cochlear implants, aneurysm clips and cardiac pacemakers. Because of the magnetic fields induced, TMS has the potential to displace the metal parts of these implants and can cause the electrical circuitry to malfunction within in the immediate vicinity $(\sim 2-10 \mathrm{~cm})$ of stimulation. While there is little data regarding the safety of patients with such devices, experts agree that that TMS poses a theoretical risk to these patients, such that they are generally excluded from TMS studies (Rossi et al., 2009). The exclusion of patients with intracranial metal or cardiac pacemakers is an especially relevant consideration in stroke patients, many of whom have 
comorbid cardiovascular disease or may have suffered complications from their stroke requiring neurovascular or neurosurgical intervention. Despite encouraging safety data on TMS, current guidelines recommend that TMS be administered in a medical setting and that there be visual monitoring during stimulation sessions. However, because the risk of seizures is extremely small, EEG monitoring during TMS is not required and is not believed to significantly increase safety (Rossi et al., 2009)

Seizure is also a theoretical concern with tDCS but far less so than for TMS. Instead, concerns principally revolve around the potential side-effects from current application to the scalp (Iyer et al., 2005). While tDCS applies direct current to the scalp and skin, it is usually applied using stimulation parameters that fall far below $\left(0.03\right.$ to $\left.0.06 \mathrm{~mA} / \mathrm{cm}^{2}\right)$ the current densities required to cause tissue damage $\left(\sim 25 \mathrm{~mA} / \mathrm{cm}^{2}\right)$ (Schlaug \& Renga, 2008). Furthermore, current tDCS protocols have not been found to induce brain edema or changes in the blood brain barrier (Nitsche et al., 2004).

Patient data confirms that tDCS only causes minimal side-effects. We recently examined 130 healthy subjects who underwent 277 tDCS sessions. During and after stimulation, patients were asked to rate sideeffects from 1 to 5 (5 being severe symptoms). We found no serious adverse effects and noted that common side-effects were tingling $(76 \%)$, itching $(68 \%)$, burning $(54 \%)$ and pain $(25 \%)$. Iyer and colleagues followed 103 patients and found no adverse effects on cognition or psychomotor measures (Iyer et al., 2005). Finally, Poreisz and colleagues (2007) examined data on 102 healthy subjects and patients who underwent a total of 567 tDCS sessions and found that mild tingling was the most common adverse effect occurring in $71 \%$ of patients. Moderate fatigue occurred in $3.5 \%$ of patients and $15 \%$ noted mild pain. Headache occurred in $5 \%$ of study subjects during the stimulation and $12 \%$ after stimulation with patients experiencing significantly more headaches. No seizures were reported in any of these studies.

\section{Conclusion}

Non-invasive brain stimulation, specifically TMS and tDCS, are emerging as promising modalities in the rehabilitation of post-stroke aphasic patients. Current data has confirmed their efficacy in the subacute and chronic stages of recovery and they may also have some efficacy in the acute stroke phase, though the data is currently quite limited. Despite the efficacy data noted above, there is much that remains to be elucidated. The neuroplastic biochemical changes that occur after stimulation and their relationship to functional recovery have yet to be fully fleshed out. Appropriate stimulation dosing and how it should be modified based on patient characteristics such as stroke size, location, and chronicity, remains incompletely explored. While the long-term efficacy of TMS has been established, it is less clear how long tDCS-induced changes actually last. Furthermore, most studies have focused on chronic non-fluent aphasic patients. More data is needed on the effects of these modalities on acute and subacute patients as well as patients with fluent aphasia. There is also little data on the unintended consequences of stimulation. Most of the safety data has only examined patients during and immediately after stimulation. However, because large networks of neurons are activated by non-invasive stimulation, it is reasonable to assume that there may be unintended downstream consequences from stimulation in patients with stroke that have not yet been identified. In order to more definitively answer persistent questions regarding the safety, efficacy, and clinical utility of noninvasive brain stimulation, future investigations will need to move beyond proof-of-principle studies toward larger scale clinical trials. Hopefully, these further investigations will bring noninvasive brain stimulation closer to becoming part of the armamentarium of tools commonly used by clinicians to treat aphasia.

\section{References}

Alexander, M.P. (1997). Aphasia: Clinical and anatomical aspects, New York: McGraw-Hill.

Ayache, S.S., Farhat, W.H., Zouari, H.G., Hosseini, H., Mylius, V. \& Lefaucheur, J.P. (2012). Stroke rehabilitation using noninvasive cortical stimulation: Motor deficit. Exp Rev of Neurotherap, 12(8), 949-972.

Aydin-Abidin, S., Trippe, J., Funke, K., Eysel, U.T. \& Benali, A. (2008). High- and low-frequency repetitive transcranial magnetic stimulation differentially activates c-Fos and zif 268 protein expression in the rat brain. Exp Brain Res, 188(2), 249261.

Baker, J.M., Rorden, C. \& Fridriksson, J. (2010). Using transcranial direct-current stimulation to treat stroke patients with aphasia. Stroke, 41, 1229-1236.

Barwood, C.H., Murdoch, B.E., Whelan, B.M., Lloyd, D., Riek, S., O’Sullivan, J.D., et al. (2010). Improved language performance 
subsequent to low-frequency rTMS in patients with chronic non-fluent aphasia post-stroke. Eur J Neurol, 18(7), 935-943.

Barwood, C.H., Murdoch, B.E., Whelan, B.M., Lloyd, D., Riek, S., O'Sullivan, J.D., et al. (2011). Modulation of N400 in chronic non-fluent aphasia using low frequency Repetitive Transcranial Magnetic Stimulation (rTMS). Brain Lang, 116(3), 125-135.

Barwood, C.H., Murdoch, B.E., Whelan, B.M., Lloyd, D., Riek, S., O'Sullivan, J.D., et al. (2012). Improved receptive and expressive language abilities in nonfluent aphasic stroke patients after application of rTMS: An open protocol case series. Brain Stimul, 5(3), 274-286.

Basso, A., Gardelli, M., Grassi, M.P. \& Mariotti, M. (1989). The role of the right hemisphere in recovery from aphasia: Two case studies. Cortex, 25, 555-566.

Basso, A. \& Marangolo, P. (2000). Cognitive rehabilitation: The emperor's new clothes? Neuropsychol Rehab, 10, 219-229.

Bhogal, S.K., Teasell, R. \& Speechley, M. (2003). Intensity of aphasia therapy, impact on recovery. Stroke, 34(4), 987-993.

Bikson, M., Datta, A. \& Elwassif, M. (2009). Establishing safety limits for transcranial direct current stimulation. Clin Neurophysiol, 120(6), 1033-1034.

Bikson, M., Datta, A., Rahman, A. \& Scaturro, J. (2010). Electrode montages for tDCS and weak transcranial electrical stimulation: Role of "return" electrode's position and size. Clin Neurophysiol, 121(12), 1976-1978.

Cappa, S.F., Perani, D., Grassi, F., Bressi, S., Alberoni, M. Franceschi, M., et al. (1997). A PET follow-up study of recovery after stroke in acute aphasics. Brain Lang, 56(1), 55-67.

Cheeran, B., Talelli, P., Mori, F., Koch, G., Suppa, A., Edwards, M., et al. (2008). A common polymorphism in the brain-derived neurotrophic factor gene (BDNF) modulates human cortical plasticity and the response to rTMS. J Physiol, 586(Pt 23), 5717-5725.

Cornelissen, K., Laine, M., Tarkiainen, A., Järvensivu, T., Martin, N. \& Salmelin, R. (2003). Adult brain plasticity elicited by anomia treatment. J Cogn Neurosci, 15, 441-461.

Crosson, B., McGregor, K., Gopinath, K.S., Conway, T.W., Benjamin, M., Chang, Y.-L., et al. (2007). Functional MRI of language in aphasia: A review of the literature and the methodological challenges. Neuropsychol Rev, 17, 157-177.

Datta, A., Elwassif, M., Battaglia, F. \& Bikson, M. (2008). Transcranial current stimulation focality using disc and ring electrode configurations: FEM analysis. J Neural Eng, 5(2), 163-174.

Fiori, V., Coccia, M., Marinelli, C.V., Vecchi, V., Bonifazi, S., Ceravolo, M.G., et al. (2011). Transcranial Direct Current Stimulation Improves Word Retrieval in Healthy and Nonfluent Aphasic Subjects. J Cogn Neurosci, 23(9), 2309-2323.

Floel, A., Meinzer, M., Kirstein, R., Nijhof, S., Deppe, M., Knecht, S., et al. (2011). Short-term anomia training and electrical brain stimulation. Stroke, 42(7), 2065-2067.

Fregni, F., Boggio, P.S., Mansur, C.G., Wagner, T., Ferreira, M.J., Lima, M.C., et al. (2005). Transcranial direct current stimulation of the unaffected hemisphere in stroke patients. Neuroreport, 16(14), 1551-1555.

Fridriksson, J. (2011). Measuring and inducing brain plasticity in chronic aphasia. J Commun Disord, 44(5), 557-563.
Fridriksson, J., Richardson, J.D., Baker, J.M. \& Rorden, C. (2011). Transcranial Direct Current Stimulation Improves Naming Reaction Time in Fluent Aphasia: A Double-Blind, ShamControlled Study. Stroke, 42(3), 819-821.

Gersner, R., Kravetz, E., Feil, J., Pell, G. \& Zangen, A. (2011). Long-term effects of repetitive transcranial magnetic stimulation on markers for neuroplasticity: Differential outcomes in anesthetized and awake animals. J Neurosci, 31(20), 75217526.

Hallett, M. (2007). Transcranial magnetic stimulation: A primer. Neuron, 55(2), 187-199.

Hamilton, R.H., Chrysikou, E.G. \& Coslett, B. (2011). Mechanisms of aphasia recovery after stroke and the role of noninvasive brain stimulation. Brain Lang, 118(1-2), 40-50.

Hamilton, R.H., Sanders, L., Benson, J., Faseyitan, O., Norise, C., Naeser, M., et al. (2010). Stimulating conversation: Enhancement of elicited propositional speech in a patient with chronic non-fluent aphasia following transcranial magnetic stimulation. Brain Lang, 113(1), 45-50.

Heiss, W.D. \& Thiel, A. (2006). A proposed regional hierarchy in recovery of post-stroke aphasia. Brain Lang, 98, 118123.

Hoogendam, J.M., Ramakers, G.M. \& Di Lazzaro, V. (2010). Physiology of repetitive transcranial magnetic stimulation of the human brain. Brain Stimul, 3(2), 95-118.

Horn, S.D., DeJong, G., Smout, R.J., Gassaway, J., James, R. \& Conroy, B. (2005). Stroke rehabilitation patients, practice, and outcomes: Is earlier and more aggressive therapy better? Arch Phys Med Rehabil, 86(12 Suppl 2), S101-S114.

Huang, Y.Z., Chen, R.S., Rothwell, J.C. \& Wen, H.Y. (2007). The after-effect of human theta burst stimulation is NMDA receptor dependent. Clin Neurophysiol, 118(5), 1028-1032.

Hummel, F., Celnik, P., Giraux, P., Floel, A., Wu, W.-H., Gerloff, C., et al. (2005). Effects of non-invasive cortical stimulation on skilled motor function in chronic stroke. Brain, $128(\mathrm{Pt} 3)$, 490-499.

Iyer, M.B., Mattu, U., Grafman, J., Lomarev, M., Sato, S. \& Wassermann, E.M. (2005). Safety and cognitive effect of frontal DC brain polarization in healthy individuals. Neurology, 64(5), 872875.

Janicak, P.G., O'Reardon, J.P., Sampson, S.M., Husain, M.M., Lisanby, S.H., Rado, J.T., et al. (2008). Transcranial magnetic stimulation in the treatment of major depressive disorder: A comprehensive summary of safety experience from acute exposure, extended exposure, and during reintroduction treatment. J Clin Psychiatry, 69(2), 222-232.

Kang, E.K., Kim, Y.K., Sohn, H.M., Cohen, L.G. \& Paik, N.J. (2011). Improved picture naming in aphasia patients treated with cathodal tDCS to inhibit the right Broca's homologue area. Restor Neurol Neurosci, 29(3), 141-152.

Karbe, H., Thiel, A. \& Weber-Luxenburger, G. (1998). Reorganization of the cerebral cortex in post-stroke aphasia studied with positron emission tomography. Neurology, 50, A321.

Khedr, E.M., Abdel-Fadeil, M.R., Farghali, A. \& Qaid, M. (2009). Role of 1 and $3 \mathrm{~Hz}$ repetitive transcranial magnetic stimulation on motor function recovery after acute ischaemic stroke. Eur J Neurol, 16(12), 1323-1330. 
Khedr, E.M., Ahmed, M.A., Fathy, N. \& Rothwell, J.C. (2005). Therapeutic trial of repetitive transcranial magnetic stimulation after acute ischemic stroke. Neurology, 65(3), 466-468.

Ko, M.H., Han, S.H., Park, S.H., Seo, J.H. \& Kim, Y.H. (2008) Improvement of visual scanning after DC brain polarization of parietal cortex in stroke patients with spatial neglect. Neurosci Lett, 448(2), 171-174.

Leger, A., Demonet, J.F., Ruff, S., Aithamon, B., Touyeras, B. \& Puel, M. (2002). Neural substrates of spoken language rehabilitation in an aphasic patient: An fMRI study. Neuroimage, 17, 174-183.

Machii, K., Cohen, D., Ramos-Estebanez, C. \& Pascual-Leone, A. (2006). Safety of rTMS to non-motor cortical areas in healthy participants and patients. Clin Neurophysiol, 117(2), 455-471.

Mansur, C.G., Fregni, F., Boggio, P.S., Riberto, M., Gallucci-Neto, J., Santos, C.M., et al. (2005). A sham stimulation-controlled trial of rTMS of the unaffected hemisphere in stroke patients. Neurology, 64(10), 1802-1804.

Medina, J., Norise, C., Faseyitan, O., Coslett, H.B., Turkeltaub, P.E. \& Hamilton, R.H. (2012). Finding the right words: Transcranial magnetic stimulation improves discourse productivity in nonfluent aphasia after stroke. Aphasiology, 26(9), 1153-1168.

Miranda, P.C., Mekonnen, A., Salvador, R. \& Ruffini, G. (2013). The electric field in the cortex during transcranial current stimulation. Neuroimage, 70, 48-58.

Monti, A., Cogiamanian, F., Marceglia, S., Ferrucci, R., Mameli, F., Mrakic-Sposta, S., et al. (2008). Improved naming after transcranial direct current stimulation in aphasia. J Neurol Neurosurg Psychiatry, 79, 451-453.

Naeser, M.A., Martin, P.I., Lundgren, K., Klein, R., Kaplan, J., Treglia, E., et al. (2010). Improved language in a chronic nonfluent aphasia patient after treatment with CPAP and TMS. Cogn Behav Neurol, 23(1), 29-38.

Naeser, M.A., Martin, P.I., Nicholas, M., Baker, E.H., Seekins, H., Helm-Estabrooks, N., et al. (2005a). Improved naming after TMS treatments in a chronic, global aphasia patient-case report. Neurocase, 11(3), 182-193.

Naeser, M.A., Martin, P.I., Nicholas, M., Baker, E.H., Seekins, H., Kobayashi, M., et al. (2005b). Improved picture naming in chronic aphasia after TMS to part of right Broca's area: An open-protocol study. Brain and Language, 93, 95-105.

Naeser, M.A., Martin, P.I., Theoret, H., Kobayashi, M., Fregni, F., Nicholas, M., et al. (2011). TMS suppression of right pars triangularis, but not pars opercularis, improves naming in aphasia. Brain Lang, 119(3), 206-213.

Naeser, M.A., Theoret, H. \& Kobayashi, M. (2002). Modulation of cortical areas with repetitive transcranial magnetic stimulation to improve naming in nonfluent aphasia. Presented at the 8th International Conference on Functional Mapping of the Human Brain.

Nitsche, M.A., Cohen, L.G., Wassermann, E.M., Priori, A., Lang, N., Antal, A., et al. (2008). Transcranial direct current stimulation: State of the art 2008. Brain Stimul, 1(3), 206-223.

Nitsche, M.A., Fricke, K., Henschke, U., Schlitterlau, A., Liebetanz, D., Lang, N., et al. (2003). Pharmacological modulation of cortical excitability shifts induced by transcranial direct current stimulation in humans. J Physiol, 553(Pt 1), 293-301.
Nitsche, M.A., Niehaus, L., Hoffmann, K.T., Hengst, S., Liebetanz, D., Paulus, W., et al. (2004). MRI study of human brain exposed to weak direct current stimulation of the frontal cortex. Clin Neurophysiol, 115(10), 2419-2423.

Nitsche, M.A. \& Paulus, W. (2000). Excitability changes induced in the human motor cortex by weak transcranial direct current stimulation. J Physiol, 527(Pt 3), 633-639.

Nitsche, M.A., Seeber, A., Frommann, K., Klein, C.C., Rochford, C., Nitsche, M.S., et al. (2005). Modulating parameters of excitability during and after transcranial direct current stimulation of the human motor cortex. J Physiol, 568(Pt 1), 291-303.

Oliveri, M. \& Caltagirone, C. (2006). Suppression of extinction with TMS in humans: From healthy controls to patients. Behav Neurol, 17(3-4), 163-167.

Oliveri, M., Rossini, P.M., Filippi, M.M., Traversa, R., Cicinelli, P., Palmieri, M.G., et al. (2000). Time-dependent activation of parieto-frontal networks for directing attention to tactile space. A study with paired transcranial magnetic stimulation pulses in right-brain-damaged patients with extinction. Brain, 123(Pt 9), 1939-1947.

Oliveri, M., Rossini, P.M., Traversa, R., Cicinelli, P., Filippi, M.M., Pasqualetti, P., et al. (1999). Left frontal transcranial magnetic stimulation reduces contralesional extinction in patients with unilateral right brain damage. Brain, 122(Pt 9), 1731-1739.

Parent, A. (2004). Giovanni Aldini: From animal electricity to human brain stimulation. Can J Neurol Sci, 31(4), 576-584.

Peterchev, A.V., Wagner, T.A., Miranda, P.C., Nitsche, M.A., Paulus, W., Lisanby, S.H., et al. (2012). Fundamentals of transcranial electric and magnetic stimulation dose: Definition, selection, and reporting practices. Brain Stimulation, 5(4), 435-453.

Poreisz, C., Boros, K., Antal, A. \& Paulus, W. (2007). Safety aspects of transcranial direct current stimulation concerning healthy subjects and patients. Brain Res Bull, 72(4-6), 208-214.

Postman-Caucheteux, W.A., Birn, R.M., Pursley, R.H., Butman, J.A., Solomon, J.M., Picchioni, D., et al. (2010). Single-trial fMRI Shows Contralesional Activity Linked to Overt Naming Errors in Chronic Aphasic Patients. J Cogn Neurosci, 22, 1299-1318.

Ranieri, F., Podda, M.V., Riccardi, E., Frisullo, G., Dileone, M., Profice, P., et al. (2012). Modulation of LTP at rat hippocampal CA3-CA1 synapses by direct current stimulation. J Neurophysiol, 107(7), 1868-1880.

Reis, J., Schambra, H.M., Cohen, L.G., Buch, E.R., Fritsch, B., Zarahn, E., et al. (2009). Noninvasive cortical stimulation enhances motor skill acquisition over multiple days through an effect on consolidation. Proc Natl Acad Sci U S A, 106(5), 1590-1595.

Roger, V.L., Go, A.S., Lloyd-Jones, D.M., Benjamin, E.J., Berry, J.D., Borden, W.B., et al. (2012). Heart disease and stroke statistics-2012 update: A report from the American Heart Association. Circulation, 125(1), e2-e220.

Rosen, H.J., Petersen, S.E., Linenweber, M.R., Snyder, A.Z., White, D.A., Chapman, L., et al. (2000). Neural correlates of recovery from aphasia after damage to left inferior frontal cortex. Neurology, 26, 1883-1894.

Rossi, C., Sallustio, F., Di Legge, S., Stanzione, P. \& Koch, G. (2013). Transcranial direct current stimulation of the affected hemi- 
sphere does not accelerate recovery of acute stroke patients. Eur J Neurol, 20(1), 202-204.

Rossi, S., Hallett, M., Rossini, P.M. \& Pascual-Leone, A. (2009). Safety, ethical considerations, and application guidelines for the use of transcranial magnetic stimulation in clinical practice and research. Clin Neurophysiol, 120(12), 2008-2039.

Rossini, P.M. \& Rossi, S. (2007). Transcranial magnetic stimulation: Diagnostic, therapeutic, and research potential. Neurology, 68(7), 484-488.

Saur, D., Lange, R. \& Baumgaertner, A. (2006). Dynamics of language reorganization after stroke. Brain, 129, 1371-1384.

Schlaug, G., Marchina, S. \& Norton, A. (2009). Evidence for plasticity in white-matter tracts of patients with chronic Broca's aphasia undergoing intense intonation-based speech therapy. Ann NY Acad Sci, 1169, 385-394.

Schlaug, G., Marchina, S. \& Wan, C.Y. (2011). The use of noninvasive brain stimulation techniques to facilitate recovery from post-stroke aphasia. Neuropsychol Rev, 21(3), 288-301.

Schlaug, G. \& Renga, V. (2008). Transcranial direct current stimulation: A noninvasive tool to facilitate stroke recovery. Expert Rev Med Devices, 5(6), 759-768.

Schrader, L.M., Stern, J.M., Koski, L., Nuwer, M.R. \& Engel, J. $\mathrm{Jr}$, (2004). Seizure incidence during single- and paired-pulse transcranial magnetic stimulation (TMS) in individuals with epilepsy. Clin Neurophysiol, 115(12), 2728-2737.

Shimizu, T., Hosaki, A., Hino, T., Sato, M., Komori, T., Hirai, S., et al. (2002). Motor cortical disinhibition in the unaffected hemisphere after unilateral cortical stroke. Brain, 125, 18961907.

Stefan, K., Kunesch, E., Benecke, R., Cohen, L.G. \& Classen, J. (2002). Mechanisms of enhancement of human motor cortex excitability induced by interventional paired associative stimulation. J Physiol, 543(Pt 2), 699-708.

Stokes, M.G., Chambers, C.D., Gould, I.C., English, T., McNaught, E., McDonald, O., et al. (2007). Distance-adjusted motor threshold for transcranial magnetic stimulation. Clin Neurophysiol, 118(7), 1617-1625.
Szaflarski, J.P., Vannest, J., Wu, S.W., DiFrancesco, M.W., Banks, C. \& Gilbert, D.L. (2011). Excitatory repetitive transcranial magnetic stimulation induces improvements in chronic poststroke aphasia. Med Sci Monit, 17(3), CR132-CR139.

Thickbroom, G.W. (2007). Transcranial magnetic stimulation and synaptic plasticity: Experimental framework and human models. Exp Brain Res, 180(4), 583-593.

Thiel, A., Habedank, B., Herholz, K., Kessler, J., Winhuisen, L., Haupt, W.F., et al. (2006). From the left to the right: How the brain compensates progressive loss of language function. Brain and Language, 98, 57-65.

Turkeltaub, P.E., Coslett, H.B., Thomas, A.L., Faseyitan, O., Benson, J., Norise, C., et al. (2012). The right hemisphere is not unitary in its role in aphasia recovery. Cortex, 48(9), 1179-1186.

Turkeltaub, P.E., Messing, S., Norise, C. \& Hamilton, R.H. (2011). Are networks for residual language function and recovery consistent across aphasic patients? Neurology, 76(20), 1726-1734.

Wagner, T., Eden, U., Fregni, F., Valero-Cabre, A., RamosEstebanez, C., Pronio-Stelluto, V., et al. (2008). Transcranial magnetic stimulation and brain atrophy: A computer-based human brain model study. Exp Brain Res, 186(4), 539-550.

Wagner, T., Fregni, F., Eden, U., Ramos-Estebanez, C., Grodzinsky, A., Zahn, M., et al. (2006). Transcranial magnetic stimulation and stroke: A computer-based human model study. Neuroimage, 30(3), 857-870.

Warburton, E., Price, C.J., Swinburn, K. \& Wise, R.J. (1999). Mechanisms of recovery from aphasia: Evidence from positron emission tomography studies. J Neurol Neuros Psychiatry, 66, 155-161.

Weiduschat, N., Thiel, A., Rubi-Fessen, I., Hartmann, A., Kessler, J., Merl, P., et al. (2011). Effects of Repetitive Transcranial Magnetic Stimulation in Aphasic Stroke: A Randomized Controlled Pilot Study. Stroke, 42(2), 409-415.

You, D.S., Kim, D.Y., Chun, M.H., Jung, S.E. \& Park, S.J. (2011). Cathodal transcranial direct current stimulation of the right Wernicke's area improves comprehension in subacute stroke patients. Brain Lang, 119(1), 1-5. 